\title{
Oxidation of NADH and NADPH by Mitochondria from the Yeast Candida utilis
}

\author{
By PETER M. BRUINENBERG, JOHANNES P. VAN DIJKEN, \\ J. GIJS KUENEN AND W. ALEXANDER SCHEFFERS* \\ Laboratory of Microbiology, Delft University of Technology, Julianalaan 67A, 2628 BC Delft, \\ The Netherlands
}

(Received 5 November 1984)

\begin{abstract}
Mitochondria were isolated from Candida utilis CBS 621 grown in carbon-limited continuous cultures on glucose, gluconate, xylose, ethanol or acetate as the carbon source and ammonia or nitrate as the nitrogen source. In all cases mitochondria were isolated which could oxidize exogenous NADH and NADPH via a cyanide- and antimycin A-sensitive but rotenoneinsensitive respiratory chain. Oxidation of NADH and NADPH was coupled to energy conservation as evidenced by high respiratory control values. Different respiratory control values of mitochondria with NADH and NADPH as well as variations in the ratio of NADH and NADPH oxidase activities indicate that separate systems exist for the oxidation of exogenous redox equivalents by mitochondria of $C$. utilis.

Variation of the NADPH requirement for biomass formation by applying different growth conditions did not result in significant changes in NADPH oxidase activities of mitochondria. It is concluded that in C. utilis NADPH can be used in dissimilatory processes for the generation of ATP.
\end{abstract}

\section{INTRODUCTION}

NADPH is an essential reductant in anabolic processes. Enzyme studies have revealed that the hexose monophosphate (HMP) pathway and possibly NADP+-linked isocitrate dehydrogenase are the major sources of NADPH in the yeast Candida utilis (Bruinenberg et al., 1983b). All available evidence indicates that the organism is unable to interconvert NADH and NADPH via transhydrogenase or analogous enzyme systems (Bruinenberg et al., 1983a, b). In theoretical calculations of the NADPH requirement for biomass formation it was demonstrated that for growth with glucose as the carbon source and ammonium as the nitrogen source, depending on the contribution of the NADP+-dependent isocitrate dehydrogenase, 2 to $7 \%$ of the glucose metabolized has to be oxidized in the HMP pathway to meet the NADPH requirement for biomass formation (Bruinenberg et al., 1983a). However, radiorespirometric studies have revealed that in C. utilis approximately 30 to $50 \%$ of the glucose is metabolized via the HMP pathway (Mian et al., 1974). Also in other yeasts (Suomalainen \& Oura, 1971) the contribution of the HMP pathway to glucose metabolism may be considerably higher than the calculated minimum.

Activities of the HMP pathway exceeding the theoretical minimum point to a mechanism for dissimilatory oxidation of NADPH. Indeed it was found that mitochondria or submitochondrial particles from several yeasts and moulds oxidize NADPH (Schuurmans Stekhoven, 1966; Djavadi et al., 1980; Schwitzguebel \& Palmer, 1981). In contrast to the situation for NADH, information on the mechanism of NADPH oxidation by yeast mitochondria is limited. It was therefore decided to study qualitative and quantitative aspects of the oxidation of NADPH by yeast mitochondria in more detail.

Mitochondrial oxidation of NADPH in catabolic processes counteracts its consumption in 
anabolic reactions. In order to study this antagonism, mitochondrial NADPH oxidation was examined under a diversity of growth conditions characterized by large differences in the anabolic NADPH requirement. This can be accomplished by cultivation of $C$. utilis on different combinations of carbon and nitrogen sources in chemostat cultures (Bruinenberg et al., 1983a,b).

In this paper an attempt is made to answer the following questions.

(1) Can mitochondria from $C$. utilis oxidize NADPH?

(2) Does this process result in ATP formation?

(3) Do NADPH oxidase activities vary in relation to growth conditions?

(4) Are NADPH and NADH oxidized via the same enzyme system?

\section{METHODS}

Micro-organism and growth conditions. Candida utilis CBS 621 was maintained on malt agar slopes. Growth conditions were as described by Bruinenberg et al. (1985). Unless mentioned otherwise, data refer to cells grown in glucose-limited chemostat culture with ammonia as the nitrogen source at a dilution rate of $0 \cdot 1 \mathrm{~h}^{-1}$. All data are the mean of two or three experiments (mean deviation 10 to $20 \%$ ).

Isolation of mitochondria. Spheroplasts were prepared and mitochondria were isolated according to the dialysis method of Bruinenberg et al. (1985).

Enzlme assa!s. Oxidase activities of intact mitochondria were assayed by measuring substrate-dependent oxygen consumption rates according to Bruinenberg et al. (1985). Substrate concentrations were: NADH, 0.25 mM : NADPH, $0.75 \mathrm{~mm}$ : ethanol, $5 \mathrm{~mm}$; pyruvate, $5 \mathrm{~mm}$; and 2 -oxoglutarate, $5 \mathrm{~mm}$. Pyruvate and 2oxoglutarate oxidase activities were determined in the presence of 1 mM-sodium malate. Respiratory control values were determined according to Chance \& Williams (1956) by adding 0.083 mm-ADP. The respiratory inhibitors rotenone and antimycin A were dissolved in isopropanol. Addition of isopropanol alone inhibited the substrate-dependent oxygen uptake by less than $10 \%$.

NADH and NADPH dehydrogenases were assayed with ferricyanide as the electron acceptor according to Bruinenberg et al. (1985). The activities in the various subcellular fractions were determined after sonication of the fractions with an MSF-150 W sonicater at 4 " $\mathrm{C}$ for $2 \mathrm{~min}$.

Orientation of the NADH and NADPH dehydrogenase activities in mitochondria was determined with the ferricyanide method of Von Jagow \& Klingenberg (1970). The external activities were assayed as described above, except that $0.65 \mathrm{M}$-sorbitol was added to the assay mixtures in order to stabilize the mitochondria. The internal activities were calculated as the difference between the activity of sonicated mitochondria and the external activity of intact organelles.

Protein was assayed according to the Lowry method with bovine serum albumin as the standard.

\section{RESULTS}

A typical outcome of an isolation of mitochondria from Candida utilis grown at $D=0 \cdot 1 \mathrm{~h}^{-1}$ with glucose as the carbon source and ammonium as the nitrogen source is presented in Table 1. NADH and NADPH oxidase activities, measured as oxygen consumption in the presence of sorbitol as the osmotic stabilizer, were totally recovered in the particulate fractions $P_{1}$ and $P_{2}$. Fraction $P_{1}$ contained well-coupled mitochondria (Fig. 1). The mitochondria oxidized both exogenous NADH and NADPH and exhibited respiratory control values of 1.8 and 1.9 , respectively. Repeated cycles of state 4 and state 3 respiration (Chance \& Williams, 1956) could be demonstrated.

The oxidation of redox equivalents produced within the mitochondria was measured as oxidase activities with either ethanol, pyruvate or 2-oxoglutarate. As is demonstrated for pyruvate in Fig. 1, high respiratory control values were obtained (see also Table 3). For none of the above-mentioned substrates could oxidase activities be detected in the soluble fraction. The oxidation of NADH and NADPH in the mitochondrial fraction $\mathrm{P}_{1}$ was completely inhibited by $\mathrm{KCN}(1 \mathrm{mM})$ and antimycin A (6 nM). Rotenone (17 nM) inhibited the oxidation of ethanol, pyruvate and 2-oxoglutarate but not the oxidation of exogenous NADH or NADPH.

The above results are in accordance with current views on the mechanism of oxidation of reduced pyridine nucleotides by fungal and plant mitochondria. In contrast to mammalian organelles, mitochondria from plants and fungi can oxidize exogenous NADH and NADPH. This oxidation is rotenone-insensitive in contrast to the intramitochondrial oxidation of reduced pyridine nucleotides (Fig. 2). In order to assess the activity of the internal NADH and NADPH 


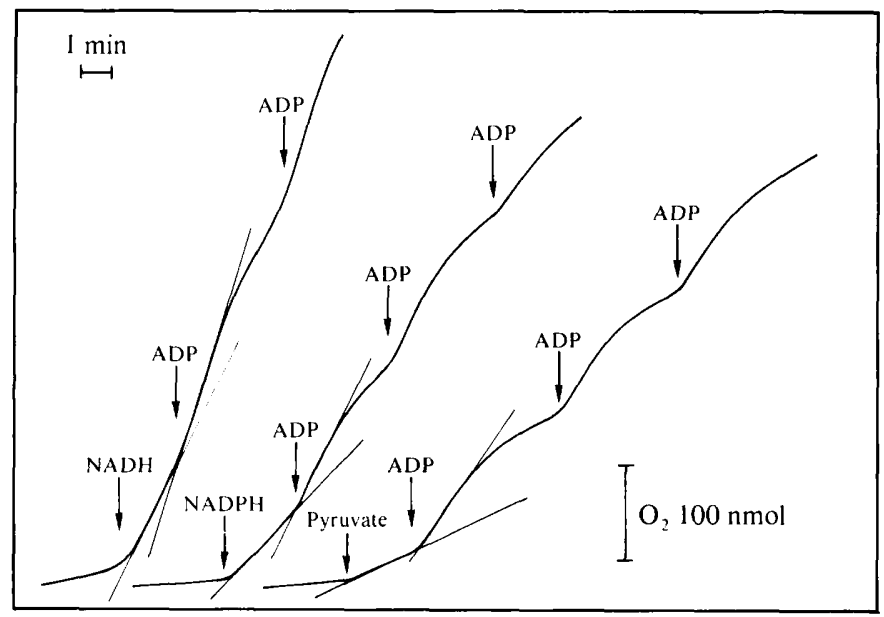

Fig. 1. Recorder tracings of oxygen consumption with NADH, NADPH or pyruvate by mitochondria of C. utilis. Respiratory control is observed after the addition of ADP.

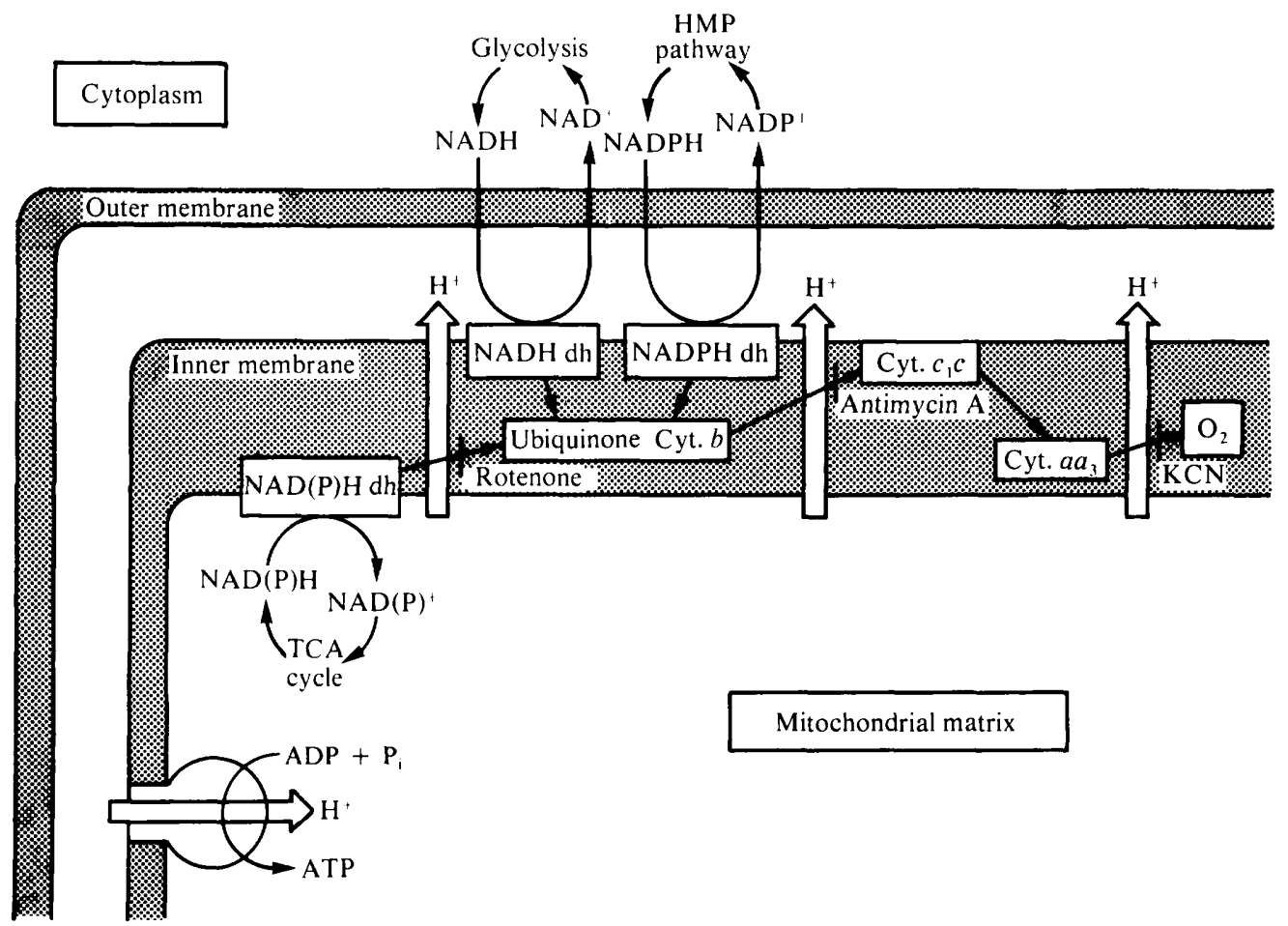

Fig. 2. Schematic representation of electron flow in yeast mitochondria from NAD(P)H to oxygen. Sites of proton translocation, of ATP synthesis and of action of respiratory inhibitors are indicated. dh. Dehydrogenase: cyt., cytochrome; HMP, hexose monophosphate.

dehydrogenase activities, mitochondria have to be sonicated since neither $\mathrm{NAD}(\mathrm{P}) \mathrm{H}$, nor ferricyanide can permeate the inner mitochondrial membrane (Von Jagow \& Klingenberg, 1970). When osmotically stabilized mitochondria were sonicated and subsequently assayed in mixtures containing sorbitol to stabilize unbroken organelles, sonication for 2 min was sufficient to unmask all NADH and NADPH dehydrogenase activities (Fig. 3). Upon sonication, the NADH dehydrogenase increased approximately 10-fold whereas NADPH dehydrogenase 


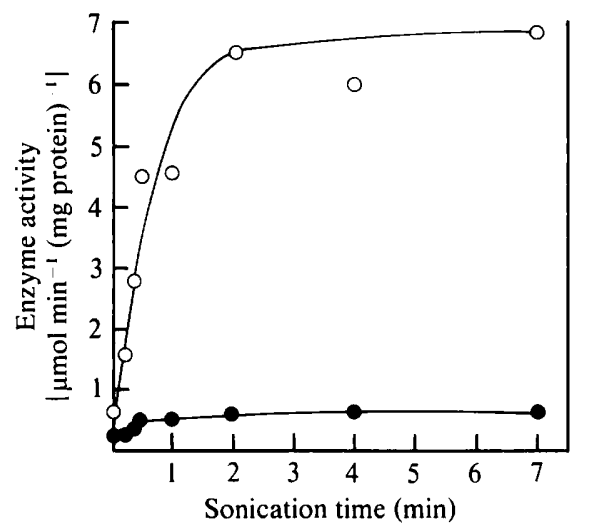

Fig. 3

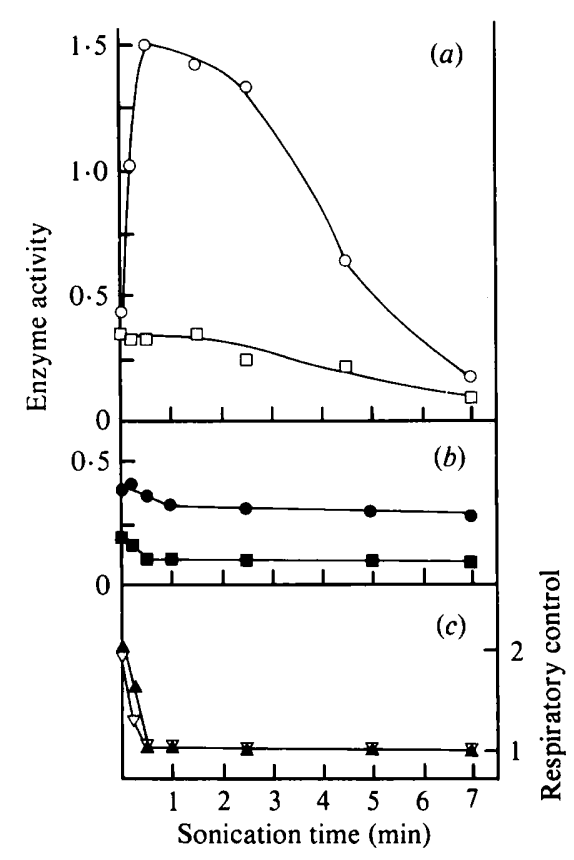

Fig. 4

Fig. 3. Release of NADH dehydrogenase $(O)$ and NADPH dehydrogenase $(O)$ after sonication of mitochondria from $C$. utilis. Samples from the suspension of mitochondria (containing $0.65 \mathrm{M}$-sorbitol) were withdrawn at intervals and assayed for NAD(P)H dehydrogenase activity in the presence of $0.65 \mathrm{M}$-sorbitol to stabilize unbroken organelles.

Fig. 4. Effect of sonication on NAD(P)H oxidase activities and respiratory control in mitochondria from $C$. utilis. (a) NADH oxidase $(O)$ and NADPH oxidase $(\square)$ in the absence of rotenone; $(b)$ NADH oxidase (O) and NADPH oxidase ( $\square)$ in the presence of rotenone; $(c)$ effect of sonication on respiratory control with $\mathrm{NADH}(\boldsymbol{\Delta})$ and $\mathrm{NADPH}(\nabla)$ in the absence of rotenone. Activities are expressed as $\mu \mathrm{mol} \mathrm{O}_{2} \mathrm{~min}^{-1}$ (mg protein) $)^{-1}$.

increased only two-fold. Unmasking of the NADH and NADPH dehydrogenases was paralleled by a sharp increase in NADH oxidase activity (Fig. $4 a$ ), which declined upon prolonging sonication for more than $30 \mathrm{~s}$. The NADPH oxidase activity exhibited a slow decrease with time. The sonication-dependent NADH oxidase activity was rotenone-sensitive (Fig. $4 b$ ), which confirms that the increase in NADH dehydrogenase activity after sonication (Fig. 3) resulted from the unmasking of the internal NADH dehydrogenase. Sonication for more than $30 \mathrm{~s}$ resulted in a complete loss of respiratory control with NADH and NADPH (Fig. 4c) although the rate of oxidation of these compounds by the mitochondria was high (compare Fig. $4 c$ and $4 a$ ).

When total NADH and NADPH dehydrogenase activities were measured in sonicated subcellular fractions, $84 \%$ of the NADH dehydrogenase but only $25 \%$ of the NADPH dehydrogenase were recovered in the mitochondrial fraction $P_{1}$ (Table 1). When, however, only external dehydrogenase activities are considered, not only the recovery of NADPH dehydrogenase, but also that of NADH dehydrogenase in the mitochondrial fraction was very low (i.e. 24 and $12 \%$ of the respective activities in fraction $\mathrm{T}$ ). This low recovery results from the much higher activity of latent, internal NADH dehydrogenase as compared to the internal NADPH dehydrogenase. It suggests that both external activities are more susceptible to solubilization during isolation than the internal activities.

Since exogenous NADPH oxidation by mitochondria would counteract NADPH consumption for cytoplasmic biosynthetic processes it was expected that the NADPH oxidase activity of mitochondria would vary under growth conditions leading to large differences in NADPH 


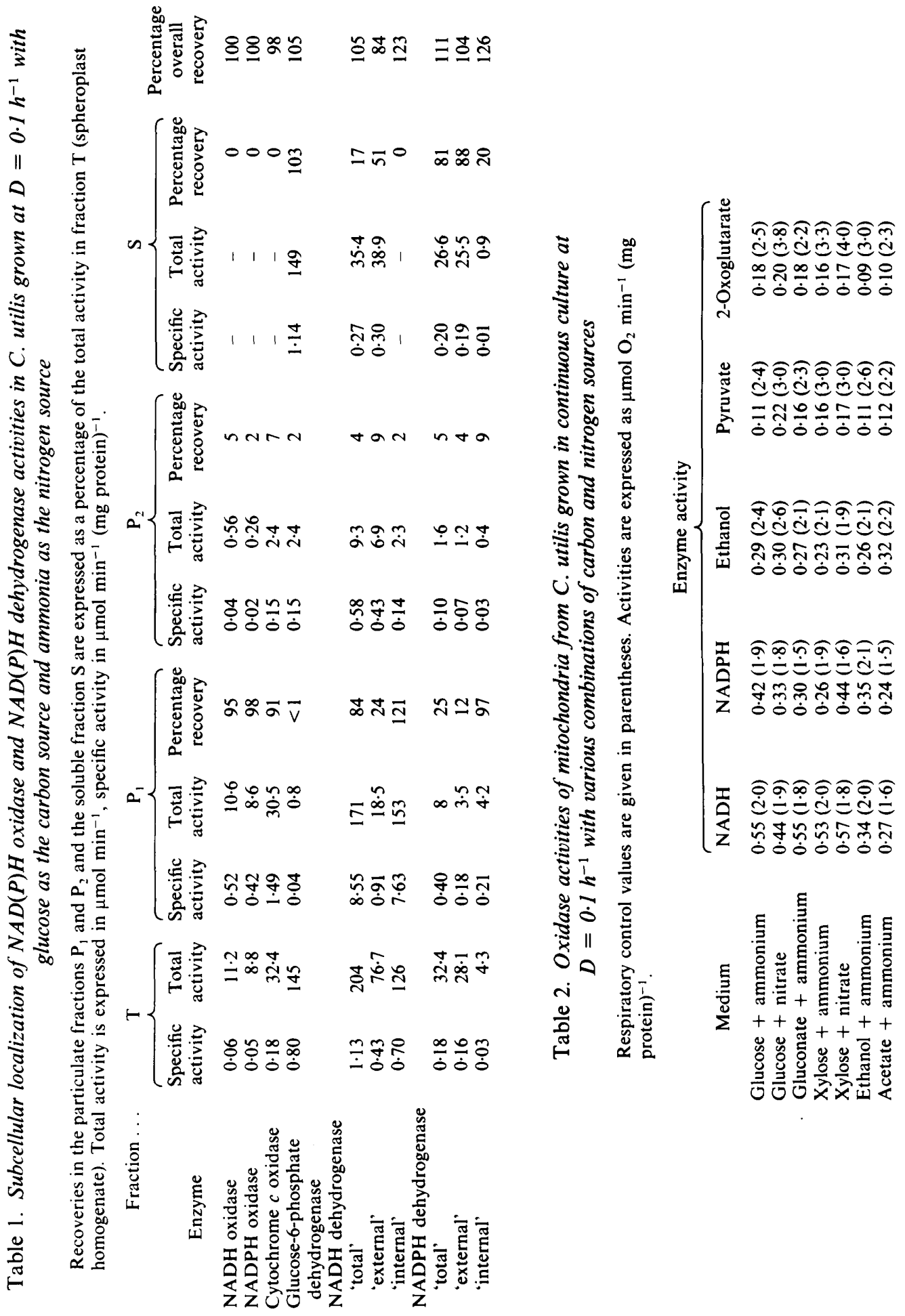




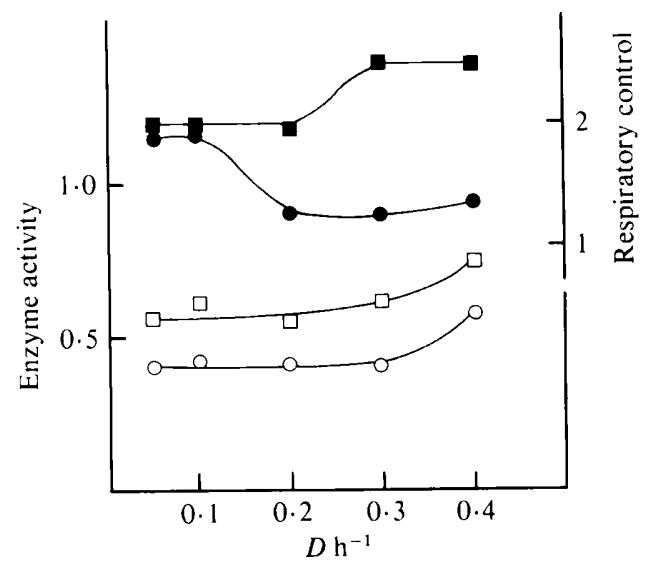

Fig. 5. NAD(P)H oxidase activities and respiratory control values with $\mathrm{NAD}(\mathrm{P}) \mathrm{H}$ of mitochondria from $C$. utilis grown in continuous culture at various dilution rates with glucose as the carbon source and ammonium as the nitrogen source. $\square$, NADH oxidase; $O$, NADPH oxidase; $\square$, respiratory control with NADH; 0 , respiratory control with NADPH. Activities are expressed as $\mu \mathrm{mol} \mathrm{O} \mathrm{min}^{-1}$ $(\mathrm{mg} \text { protein })^{-1}$.

requirement for anabolic processes. The results presented in Table 2 reveal, however, that the variation in NADPH oxidase activities does not correspond with the NADPH requirement for biomass formation. For instance, during growth on xylose plus nitrate, which provokes the highest theoretical NADPH requirement (Bruinenberg et al., 1983a), mitochondria had an NADPH oxidase activity similar to that during growth on glucose plus ammonium.

The ratio of external NADH and NADPH oxidase activities varied with the growth conditions (Table 2). In contrast to growth with glucose, xylose or gluconate, which resulted in somewhat higher NADH oxidase activities as compared with NADPH oxidase, growth with ethanol or acetate led to approximately the same activities for both oxidases (Table 2). Also the oxidase activities with ethanol, pyruvate or 2-oxoglutarate varied in an unexpected manner with the growth conditions. Despite the high activity of the TCA cycle during growth on ethanol or acetate, 2-oxoglutarate oxidase activity of mitochondria from cells grown with these substrates was lower than for growth with the other carbon sources. Furthermore, although pyruvate dehydrogenase has no apparent role during growth with ethanol and acetate, relatively high pyruvate oxidase activities in mitochondria from cells grown with these carbon sources were detectable. For all growth conditions tested mitochondria exhibited respiratory control (Table 2) with reduced pyridine nucleotides, ethanol, pyruvate or 2-oxoglutarate. Oxidase activities were hardly affected by the growth rate, as is demonstrated in Fig. 5 for NADH and NADPH oxidase. However, with increasing dilution rates the respiratory control with NADPH decreased, in contrast to that with NADH (Fig. 5).

\section{DISCUSSION}

The results presented above clearly demonstrate that mitochondria from Candida utilis grown in carbon-limited continuous culture exhibit cyanide- and antimycin A-sensitive oxidation of exogenous NADPH. Furthermore, the occurrence of respiratory control reveals that oxidation of NADPH may generate energy in the form of ATP. This is in contrast to oxidation by the socalled alternative respiration systems, which are cyanide- and antimycin A-insensitive and nonphosphorylating (Lloyd \& Edwards, 1978).

Oxidation of exogenous NADH and NADPH seems a general property of mitochondria from plants and fungi (Schuurmans Stekhoven, 1966; Schwitzguebel \& Palmer, 1981; Palmer \& Møller, 1982). Plants have systems for the external oxidation of NADH and NADPH that differ in sensitivity to chelators and cations (Møller \& Palmer, 1981). In mitochondria from C. utilis similar effects were not detectable (results not shown), but the variation in the ratio of NADH 
and NADPH oxidase activities as well as the variation in the respiratory control values (Fig. 5) indicates that two systems exist for the oxidation of exogenous NADH and NADPH by mitochondria in this yeast.

At present, it is not possible to attribute the oxidation of cytoplasmic NADH in yeasts exclusively to the external NADH oxidase. Various shuttle mechanisms, such as the ethanolacetaldehyde shuttle (Von Jagow \& Klingenberg, 1970) may contribute to mitochondrial NADH oxidation in vivo. For oxidation of cytoplasmic NADPH, however, the operation of the external oxidase is presently the only conceivable possibility. In addition, it may be noted that the NADH and NADPH oxidase activities of the mitochondrial preparations from C. utilis (Table 2) far exceed the actual requirements for growth on glucose. It can be calculated that for growth of the organism in a glucose-limited chemostat culture with ammonium as the nitrogen source at $D=0 \cdot 1 \mathrm{~h}^{-1}$, a minimal activity of $0.04 \mu \mathrm{mol} \mathrm{O}_{2} \mathrm{~min}^{-1}$ (mg protein $)^{-1}$ is required to oxidize the $\mathrm{NADH}$ generated in the cytoplasm during dissimilatory and assimilatory processes. It would therefore appear that, at least for growth on glucose, shuttle mechanisms for oxidation of cytoplasmic reduced pyridine nucleotides are not indispensable.

Most of the NADH dehydrogenase activity was located on the inside of the mitochondrion (Table 1, Fig. 2), whereas the NADPH dehydrogenase activity was about equally distributed over the inside and outside of the mitochondrial membrane. According to Tottmar \& Ragan (1971) and Mackler et al. (1981) the internal NADH dehydrogenase in yeasts cannot react, with NADPH. Hence the existence of internal NADPH dehydrogenase activity in $C$. utilis suggests that inside the mitochondrion two distinct dehydrogenases may be present for the oxidation of NADH and NADPH. The external NADH dehydrogenase is reported to be active with both NADPH and NADH (Mackler et al., 1980). However, as mentioned above, the observed variations in external NADH and NADPH oxidase activities (Table 2, Fig. 5) indicate that, as in plants, two separate systems are responsible for external oxidation of these coenzymes in $C$. utilis.

The NADPH-producing enzymes of the hexose monophosphate pathway, among others glucose-6-phosphate dehydrogenase, are located in the cytoplasm (Table 1). Also, NADP+linked isocitrate dehydrogenase was recovered at more than $90 \%$ in the soluble fraction (results not shown). Since the inner mitochondrial membrane is impermeable to pyridine nucleotides (Von Jagow \& Klingenberg, 1970), redox equivalents must be oxidized in the cell compartments where they are produced. In view of the cytoplasmic localization of NADPH-producing systems, the external NADPH oxidase activity may be quantitatively more important than the internal NADPH oxidase.

Even with the gentle method used for isolation of mitochondria considerable amounts of both NADH and NADPH dehydrogenase activities were detected in the soluble fraction (Table 3). For other yeasts grown in batch cultures similar observations have been made (Perlman \& Mahler, 1970; Cartledge \& Lloyd, 1972; Delaissé et al., 1981). Without an electron-accepting system it is difficult to envisage a physiological role for the NADH and NADPH dehydrogenases in the soluble fraction of the cell. A possible electron-accepting system for the NADPH dehydrogenase in the cytoplasm such as cytochrome P-450 is only present in yeasts under fermentative conditions (Kärenlampi et al., 1981; Trinn et al., 1982) or during $n$-alkane metabolism (Tanaka et al., 1982). Under our experimental conditions cytochrome P-450 could not be detected in $C$. utilis. Clearly, in this organism NADPH dehydrogenase activity is associated, at least in part, with the mitochondria.

The oxidative capacities of mitochondria isolated from C. utilis were relatively unaffected by the carbon and nitrogen source used for growth. Significantly lower NADPH oxidase activities of mitochondria, expected under growth conditions with a high anabolic NADPH consumption, for example during growth with xylose plus nitrate, were not encountered (Table 2).

The activities of the enzymes of the main NADPH-producing pathway (the hexose monophosphate pathway) varied in accordance with the anabolic NADPH requirement (Bruinenberg et al., 1983a,b). Hence it seems likely that, when growth conditions require adjustment of enzyme activities for the maintenance of the NADPH balance in the cell, this occurs at the level of NADPH production and not of mitochondrial oxidation of NADPH. 
The possible involvement of the respiratory chain in the reoxidation of NADPH in yeasts has been an outstanding problem for many decades. After the discovery of NADPH, produced in the 'Zwischenferment' (glucose-6-phosphate dehydrogenase) reaction, it was suggested that this redox equivalent was the coupling factor between glucose breakdown and respiration (Haas et al., 1940; Horecker, 1965, 1978). This view was abandoned after the discovery that NADH and not NADPH acts as the substrate in oxidative phosphorylation (Lehninger, 1951). Although further radiorespirometric studies on the contribution of the hexose monophosphate pathway are needed to elucidate the quantitative importance of dissimilatory NADPH oxidation in yeasts, our results with Candida utilis indicate that the original views on the role of NADPH in respiration deserve reconsideration.

We thank Hans J. van der Hoek for skilled technical assistance.

\section{REFERENCES}

Bruinenberg, P. M., van Dijken, J. P. \& Scheffers, W. A. (1983a). A theoretical analysis of NADPH production and consumption in yeasts. Journal of General Microbiology 129, 953-964.

Bruinenberg, P. M., VAN DiJken, J. P. \& SchefFers, W. A. (1983b). An enzymic analysis of NADPH production and consumption in Candida utilis. Journal of General Microbiology 129, 965-971.

Bruinenberg, P. M., van Dijken, J. P., Kuenen, J. G. \& SchefFers, W. A. (1985). Critical parameters in the isolation of mitochondria from Candida utilis grown in continuous culture. Journal of General Microbiology 131, 1035-1042.

Cartledge, T. G. \& Lloyd, D. (1972). Subcellular fractionation by differential and zonal centrifugation of aerobically grown glucose-derepressed Saccharomyces carlsbergensis. Biochemical Journal 126, 381-393.

Chance, B. \& Williams, G. R. (1956). The respiratory chain and oxidative phosphorylation. Advances in Enzymology 17, 65-134.

Delaissé, J. M., Martin, P., Verheyen-Bouvy, M. F. \& NiJns, E. J. (1981). Subcellular distribution of enzymes in the yeast Saccharomycopsis lipolytica, grown on $n$-hexadecane, with special reference to the $\omega$-hydroxylase. Biochimica et biophysica acta 676, 7790

Duavadi, F. H. S., Moradi, M. \& Duavadi-OhanIANCE, L. (1980). Direct oxidation of NADPH by submitochondrial particles from Saccharomyces cerevisiae. European Journal of Biochemistry 107, 501504.

HaAs, E., Horecker, B. L. \& Hogness, T. R. (1940). The enzymatic reduction of cytochrome c. Cytochrome c reductase. Journal of Biological Chemistry 136, 747-774.

HORECKeR, B. L. (1965). Pathways of carbohydrate metabolism and their physiological significance. Journal of Chemical Education 42, 244-253.

HoreCKer, B. L. (1978). Yeast enzymology: retrospectives and perspectives. In Biochemistry and Genetics of Yeasts. Pure and Applied Aspects, pp. 1-15. Edited by M. Bacila, B. L. Horecker \& A. O. M. Stoppani. New York, San Francisco: Academic Press.

Kärenlampi, S. O., Marin, E. \& Hänninen, O. O. P. (1981). Effect of carbon source on the accumulation of cytochrome $P-450$ in the yeast Saccharomyces cerevisiae. Biochemical Journal 194, 407-413.
LEHNINGER, A. L. (1951). Oxidative phosphorylation in diphosphopyridine nucleotide-linked systems. In Phosphorus Metabolism. A Symposium on the Role of Phosphorus in the Metabolism of Plants and Animals, vol. I, pp. 344-366. Edited by W. D. McElroy \& B. Glass. Baltimore: The Johns Hopkins Press.

LloYd, D. \& EDWARDS, S. W. (1978). Electron transport pathways alternative to the main phosphorylating respiratory chain. In Functions of Alternatice Terminal Oxidases. Proceedings of the $11 \mathrm{th}$ FEBS Meeting, pp. 1-10. Edited by H. Degn, D. Lloyd \& G. C. Hill. Oxford, New York: Pergamon Press.

Mackler, B., Haynes, B., Person, R. \& Palmer, G. (1980). Electron transport systems of Candida utilis. Purification and properties of the respiratory chainlinked external NADH dehydrogenase. Biochimica et biophysica acta 591, 289-297.

Mackler, B., Bevan, C., Person, R. \& Davis, K. A. (1981). Purification and properties of the respiratory chain-linked internal NADH dehydrogenase of Candida utilis. Biochemistry International 3, 9-17.

Mian, F. A., Fencl, Z., Prokop, A., Mohagheghi, A. \& FAZELI, A. (1974). Effect of growth rate on the glucose metabolism of yeast grown in continuous culture. Radiorespirometric studies. Folia microbiologica 19, 191-198.

Møller, I. M. \& Palmer, J. M. (1981). The inhibition of exogenous NAD $(\mathrm{P}) \mathrm{H}$ oxidation in plant mitochondria by chelators and mersalyl as a function of pH. Physiologia plantarum 53, 413-420.

Palmer, J. M. \& Møller, I. M. (1982). Regulation of $\mathrm{NAD}(\mathrm{P}) \mathrm{H}$ dehydrogenases in plant mitochondria. Trends in Biochemical Sciences 7, 258-261.

Perlman, P. S. \& Mahler, H. R. (1970). Intracellular localization of enzymes in yeast. Archives of Biochemistry and Biophysics 136, 245-259.

Schuurmans Stekhoven, F. M. A. H. (1966). Studies on yeast mitochondria. I. Existence of three phosphorylation sites along the respiratory chain of isolated yeast mitochondria. Archives of Biochemistry and Biophysics 115, 555-568.

Schwitzguebel, J. P. \& Palmer, J. M. (1981). Properties of mitochondria isolated from Neurospora crassa grown with acetate. FEMS Microbiology Letters 11, 273-277.

Suomalainen, H. \& OURa, E. (1971). Yeast nutrition and solute uptake. In The Yeasts, vol. 2, pp. 3-74. 
Edited by A. H. Rose \& J. S. Harrison. London \& New York: Academic Press.

TanaKa, A., Osumi, M. \& Fukui, S. (1982). Peroxisomes of alkane-grown yeast: fundamental and practical aspects. Annals of the New York Academy of Sciences 386, 183-199.

Tottmar, S. O. C. \& Ragan, C. I. (1971). The purification and properties of the respiratorychain reduced nicotinamide-adenine dinucleotide dehydrogenase of Torulopsis utilis. Biochemical Journal 124, 853-865.
TrinN, M., Käppeli, O. \& Fiechter, A. (1982). Occurrence of cytochrome $\mathrm{P} 450$ in continuous cultures of Saccharomyces cerevisiae. European Journal of Applied Microbiology and Biotechnology 15, 6468.

Von Jagow, G. \& K Lingenberg, M. (1970). Pathways of hydrogen in mitochondria of Saccharomyces carlsbergensis. European Journal of Biochemistry 12 , 583-592. 